\title{
Quantitative measurement of hopper flow using MRI
}

\author{
Maral Mehdizad $^{1 *}$, Luke Fullard ${ }^{2}$, Petrik Galvosas ${ }^{3}$ and Daniel Holland ${ }^{1}$ \\ ${ }^{1}$ Department of Chemical and Process Engineering, University of Canterbury, New Zealand \\ ${ }^{2}$ School of Fundamental Sciences, Massey University, New Zealand \\ ${ }^{3}$ MacDiarmid Institute for Advanced Materials and Nanotechnology, School of Chemical and Physical Sciences, Victoria University, \\ New Zealand
}

\begin{abstract}
To gain fundamental insight into granular flow behaviour, quantitative measurements of velocity and solid fraction are required. The aim of this study is to measure the solid fraction and velocity of 3D granular flows quantitively using a recently developed MRI method. Time-averaged spatial maps of the solid fraction and velocity are obtained for hoppers with wall angles of $30^{\circ}, 60^{\circ}$, and $90^{\circ}$. From these maps, the mass flow rate of the material was calculated along the height of the hoppers. Excellent agreement was observed between the MRI and gravimetric mass flow rate measurements, confirming the quantitative nature of the measurements. The resulting solid fraction and velocity measurements provide insight into the dynamics of granular flow.
\end{abstract}

\section{Introduction}

A better understanding of granular rheology requires measurements of the solid fraction and velocity, which are challenging due to their opacity. To date, the solid fraction and velocity of granular flows are most commonly measured using high speed cameras [1]. These measurements are restricted to wall observations and do not provide any information about the internal dynamics of three-dimensional flows. Threedimensional flows can be measured using x-ray or electrical tomographic methods [2]. However, these techniques only provide density measurements and do not allow for velocity measurements. Positron Emission Particle Tracking (PEPT) [3] allows for 3D measurements of velocity and solid fraction but requires the assumption of ergodicity, which may not hold for granular flows. Magnetic Resonance Imaging (MRI) has been used to study optically opaque granular flows in three dimensions making it a powerful alternative to other experimental techniques. Here we demonstrate accurate quantitative measurements of solid fraction and velocity in three-dimensional hoppers using MRI.

MRI measurements of granular flow are mostly of velocity only [4]. The few solid fraction measurements that have been presented are either of static samples $[5,6]$, or do not provide sufficient accuracy $[7,8]$ to provide insight into the granular rheology. Quantitative MRI measurements of solid fraction during flow are challenging because the particles motion during imaging affects the measured signal intensity. Recently, we developed an advanced MRI method and demonstrated that the solid fraction of granular flows can be measured quantitatively with a precision of about $1 \%$ [9].
The purpose of this paper is to use the recently developed solid fraction measurement technique [9], in conjunction with quantitative velocity measurements, to characterize the solid fraction and velocity of granular flow through additively manufactured (3D-printed) hoppers. We demonstrate that the measurements are quantitative by calculating the mass flow rate from the MRI results and comparing this with gravimetric measurements. Using these measurements, we examine the effect of hopper angle on velocity and solid fraction.

\section{Experimental set-up}

The studied geometry was a cylindrical hopper with an inner diameter of $24 \mathrm{~mm}$. The diameter of the outlet of the hopper was $9.5 \mathrm{~mm}$. Three hoppers of the same diameter and outlet size but with wall angles $(\theta)$ of $30^{\circ}$, $60^{\circ}$, and $90^{\circ}$ were studied. The schematic design of the $30^{\circ}$ hopper is shown in Figure 1. The enclosed area at the bottom of the hopper, called the "pouch" is used to contain static particles that provide a reference signal to ensure that the measurement of solid fraction is quantitative [10]. Brown mustard seeds of diameter $d_{\mathrm{p}}=1.3 \mathrm{~mm}$ and particle density of $1140 \mathrm{~kg} \mathrm{~m}^{-3}$ were studied. Mustard seeds are used as they are nearly spherical and contain oil which is what is detected during the MRI measurement. The hopper system was kept filled with particles to ensure that the flow rate is constant and independent of height [11].

All images were obtained using a $9.4 \mathrm{~T}$ superconducting magnet with ${ }^{1} \mathrm{H}$ resonance frequency of $400 \mathrm{MHz}$ linked to a Bruker Avance I spectrometer. Radio frequency transmission and reception were performed by a $30 \mathrm{~mm}$ diameter birdcage coil. A 3 -axis

\footnotetext{
* Corresponding author: maral.mehdizad@pg.canterbury.ac.nz

A video is available at https://doi.org/10.48448/jsys-4q74
} 
shielded gradient set capable of producing a maximum gradient strength of $1.51 \mathrm{~T} \mathrm{~m}^{-1}$ was used for imaging.
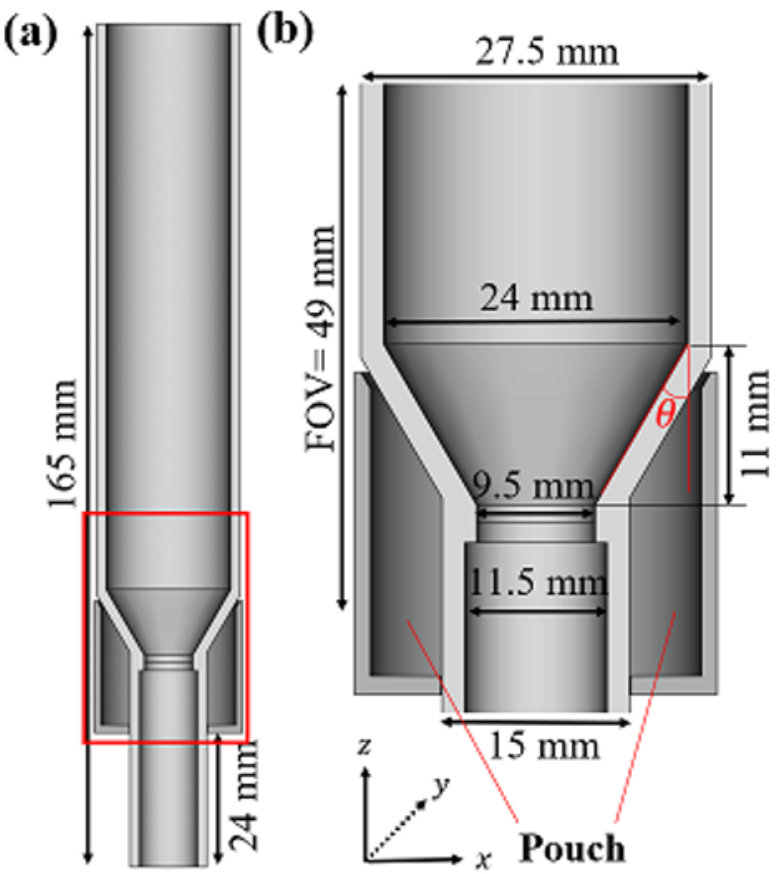

Fig. 1. (a) The schematic design of the $30^{\circ}$ hopper. (b) A close-up of the imaged part of the hopper.

As we have recently shown, the signal intensity in an MRI measurement is directly proportional to the solid fraction, provided the measurements are referenced to an appropriate internal standard and corrected for radiofrequency (r.f.) homogeneity using an appropriate reference image $I_{\text {ref }}$ [9]. To calibrate the measurements, the solid fraction was measured by filling the hoppers with a known mass of particles. For each hopper angle, 15 static samples were measured at different packing state. Assuming that the solid fraction in the hopper is uniform, the average solid fraction is obtained from:

$$
\phi_{\text {seed }}=V_{\text {seed }} / V_{\text {total }}
$$

where $V_{\text {seed }}$ is the total volume of seeds in the hopper, and $V_{\text {total }}$ is the total volume of the hopper that is occupied by seeds (i.e. including void spaces between the seeds). $V_{\text {seed }}$ is determined by dividing the weight of the seeds by their measured particle density. $V_{\text {total }}$ is determined by measuring the height of the particles in the hopper. The volume was then determined from a calibration obtained by filling the hoppers with water. For any given amount of seeds, the height of the bed could vary up to about $5 \mathrm{~mm}$ based on its packing state.

Spatial maps of the solid fraction were extracted from 3D images of the hoppers obtained by using a sectoral-SPRITE pulse sequence [12] with 110 cones and $1352 \mathrm{rf}$ pulses in each cone. This method is suitable for materials containing hydrogen nuclei with $T_{2}^{*}>$ $0.1 \mathrm{~ms}$, such as oil-filled pharmaceutical particles or many types of seeds. The flip angle of the r.f. pulses was set to $13^{\circ}$. The phase encoding time was $100 \mu$ s and the repetition time was $2 \mathrm{~ms}$. The delay between cones was chosen to be $3.5 \mathrm{~s}$. The maximum gradient strength was
$0.25 \mathrm{~T} \mathrm{~m}^{-1}$ with a spatial resolution of $0.8 \mathrm{~mm}$ in the vertical direction and $0.5 \mathrm{~mm}$ in the transverse directions. The total acquisition time for one image (2 scans) was $24 \mathrm{~min}$. The final images were obtained by averaging the signal from three individual images.

Spatial maps of the time-averaged vertical velocity of particles were obtained using a slice selective PulsedGradient Spin Echo (PGSE) imaging method [4]. Experiments were performed using a flow-encoding gradient with a maximum strength of $0.62 \mathrm{~T} \mathrm{~m}^{-1}$ and a width of $0.15 \mathrm{~ms}$ incremented in 9 steps. The observation time was $1.4 \mathrm{~ms}$. The field of flow was 0.8 $\mathrm{m} \mathrm{s}^{-1}$. The field of view was $30 \mathrm{~mm}$ in the horizontal direction and $49 \mathrm{~mm}$ in vertical direction. The total acquisition time was about $1 \mathrm{~h}$.

\section{Results and discussion}

\subsection{Signal correction procedure}

It is necessary to calibrate the images to convert the signal intensity to a quantitative solid fraction. For this conversion, the average solid fraction was measured gravimetrically for 45 samples with solid fractions between 0.55 and 0.65 . Each of these samples was then imaged to determine a corresponding signal intensity. The average signal intensity from a $7.5 \mathrm{~mm}$ by $7.5 \mathrm{~mm}$ by $15 \mathrm{~mm}$ region of interest in the centre of the image was determined. The resulting calibration of the signal intensity is shown in Figure 2. The vertical error bars represent the combined uncertainty from the standard error arising from the signal of the internal standard and from the image intensity in the region of interest. There is a linear correlation $\left(\mathrm{R}^{2}=0.999\right)$ between the signal intensity and the solid fraction. There is only a $0.5 \%$ residual variation in the estimated solid fraction, which is within the experimental uncertainty. The error associated with the measurement of the image intensity is $0.6 \%$ (based on signal-to-noise ratio). This means we anticipate a root sum of squared error of $0.8 \%$ in any pixel of the solid fraction map $\left(\overline{I / I_{\text {ref }}}\right)$.

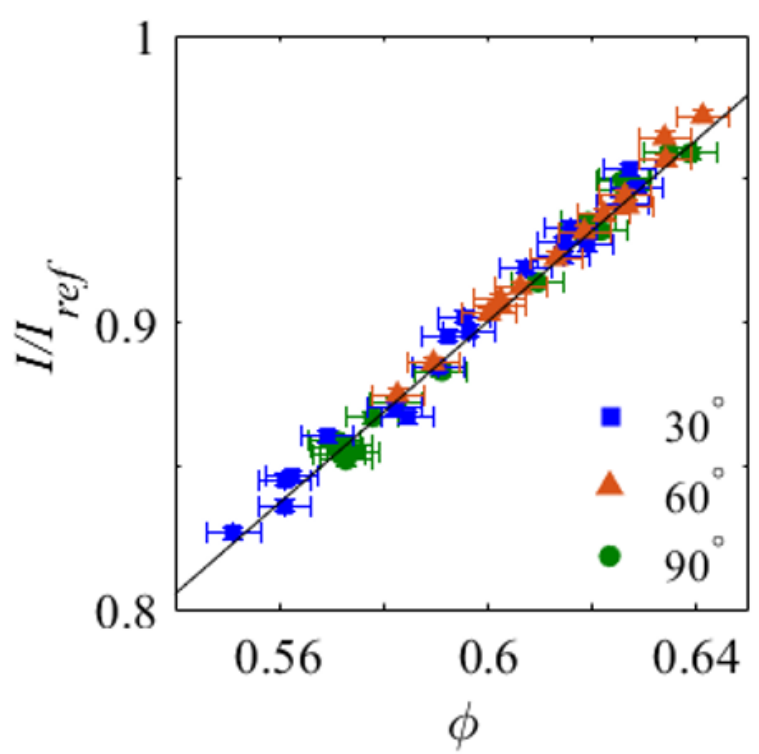

Fig. 2. Normalized signal intensity vs solid fraction. 


\subsection{Solid fraction}

Time-averaged solid fraction maps of the hoppers during flow were produced by applying the calibration curve, shown in Figure 2, to the obtained 3D images of the hoppers. Figure 3 shows the radially averaged solid fraction map of the $30^{\circ}$ hopper during steady flow. The bright spots at the wall in the sloped region arise from a slight misalignment between the calibration image and the solid fraction image and should be ignored. The solid fraction decreases from the top of the hopper towards the outlet. As shown in Figure 4, three different flow behaviours are observed in the cylindrical, conical, and outlet sections of the hopper. In the cylindrical part of the hopper $(\mathrm{z}=20 \mathrm{~mm})$, the solid fraction is at its maximum and is approximately constant along the radius but begins to decrease about $5 \mathrm{~mm}\left(4 d_{\mathrm{p}}\right)$ from the wall. From the top of the conical part $(\mathrm{z}=6 \mathrm{~mm})$ of the hopper to the outlet $(\mathrm{z}=0 \mathrm{~mm})$, the solid fraction is nearly constant along the radius, but it starts to increase about $1 d_{\mathrm{p}}$ away from the wall. This may simply be due to the misalignment of the reference image, but it may also arise from an ordering effect at the wall. Below the outlet $(\mathrm{z}=-2 \mathrm{~mm})$, the solid fraction has its maximum value at the centre and reduces towards the edge of the flow. For the $60^{\circ}$ and $90^{\circ}$ hoppers, qualitatively similar behaviour was observed. In the $90^{\circ}$ hopper that has no angled wall, a region of relatively high solid fraction (equal to wall solid fraction of $30^{\circ}$ and $60^{\circ}$ hoppers) was observed at the bottom corners of the $90^{\circ}$ hopper.

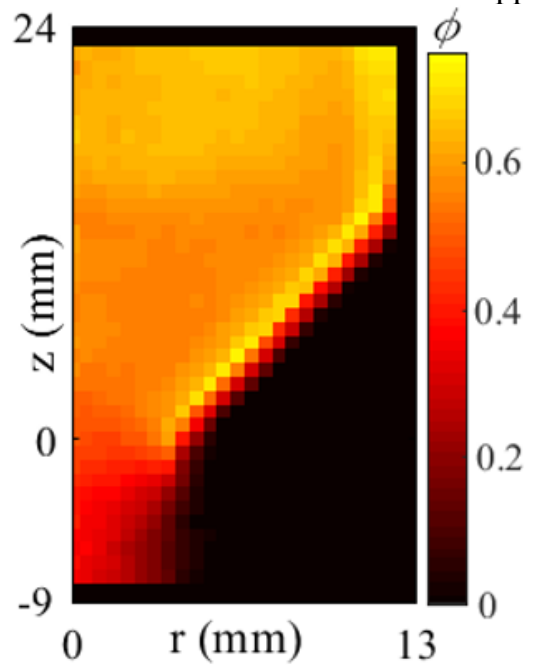

Fig. 3. The radially averaged solid fraction map of the $30^{\circ}$ hopper during steady flow.

Figure 5 shows a quantitative comparison of the solid fraction along the centreline (two central pixels are averaged) of the hoppers with angles of $30^{\circ}, 60^{\circ}$, and $90^{\circ}$. The origin of the $\mathrm{z}$-coordinate is located at the outlet of the hoppers. The bulk solid fraction at the top part of the hoppers is the same for all three hoppers and around the expected close packing limit of 0.64. At about $\mathrm{z}=15 \mathrm{~mm}$, the solid fraction decreases to about 0.57 . The location of the decrease is dependent on the hopper angle. The solid fraction starts to decrease closer to the outlet for hoppers with smaller hopper angles. The solid fraction of all three hoppers is approximately constant at a value of 0.57 from $\mathrm{z}=11 \mathrm{~mm}$ to $\mathrm{z}=4 \mathrm{~mm}$. At about $4 \mathrm{~mm}$ above the outlet, the solid fraction starts to decrease again towards the outlet. At the centre of the outlet, the solid fraction decreases with increasing the hopper angle.

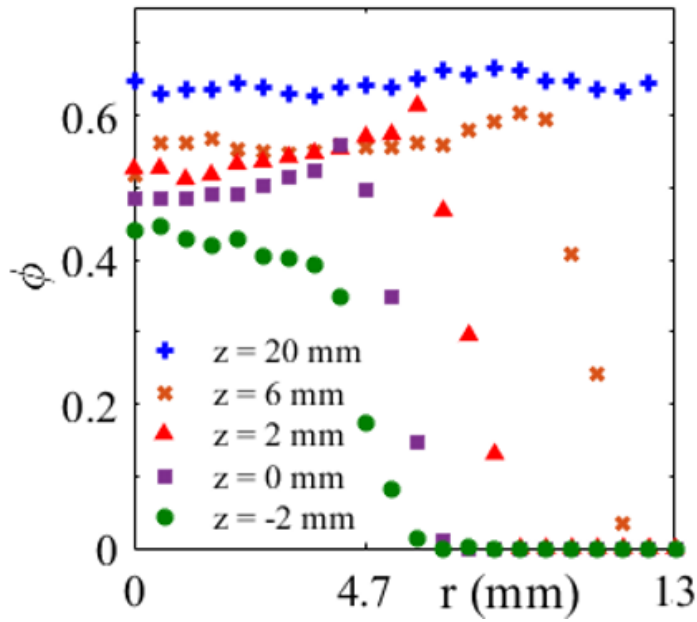

Fig. 4. The solid fraction profiles along the radius of the $30^{\circ}$ hopper.

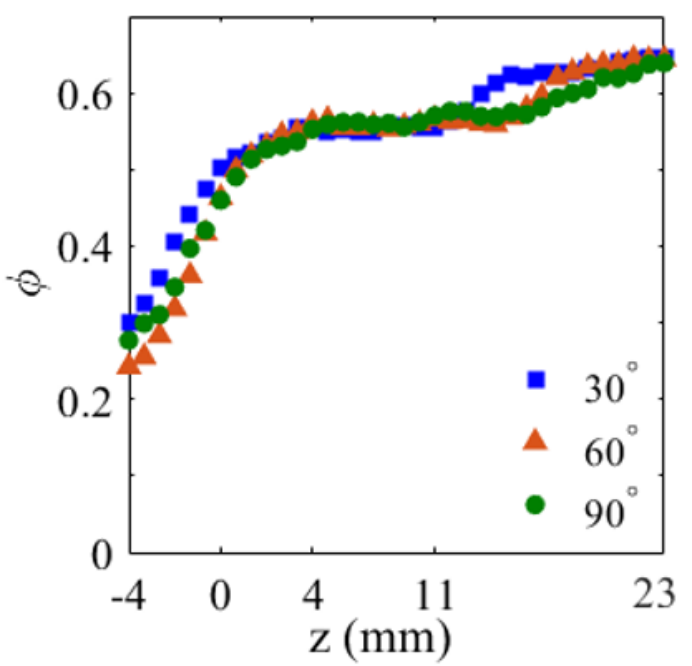

Fig. 5. The solid fraction profiles along the centreline of the hoppers.

\subsection{Velocity}

Figure 6 shows a vertical 2D map of the mean vertical velocity of the particles, which displays a good symmetry around the centreline. The velocity of the particles was not captured from $2 \mathrm{~mm}$ below the outlet because particles are moving too fast to be captured accurately. A mass flow was observed in the $30^{\circ}$ hopper as all the particles at different locations of the hopper were in motion. In $60^{\circ}$ and $90^{\circ}$ hoppers, a funnel flow was observed as there were some stagnant zones at the bottom corners of the hoppers. In the cylindrical part of the hopper at the top of the image, the vertical velocity is approximately constant along the height and along the radius. In this region where the solid fraction is the highest, the vertical velocity of the particles is the lowest and nearly the same for all three hoppers. For all three hoppers, the vertical velocity starts to increase towards the outlet from about $11 \mathrm{~mm}$ above the outlet, which is where the $30^{\circ}$ hopper begins to taper. At any given height in this region, vertical velocity has its maximum value at the centre and reduces towards the wall. 


\subsection{Flow rate}

To confirm that the solid fraction and velocity maps are quantitative, a mass flow rate map was generated. The total mass flow rate at each vertical position was obtained from:

$$
\dot{m}=\rho_{s} \sum \phi_{i} v_{i} A_{i}
$$

where $\phi_{i}$ is the solid fraction, $v_{i}$ is the velocity, and $A_{i}$ is the area of each voxel, and the summation is over all voxels at a given vertical position. The velocity maps were only measured in two dimensions. Therefore, the area used in Eq. 2, corresponds to an annular region of the width of the voxel at a given radial position, assuming cylindrical symmetry. Figure 7 shows the integrated flow rate through the cross-sectional area of the hopper at each vertical position within the hopper. The integrated mass flow rate is constant along the height of the hopper, as is expected based on conservation of mass. The integrated flow rate starts to decrease below the outlet, indicating the quantitative accuracy of the measurement begins to decrease here. This decrease occurs because faster moving particles may leave the MRI system leading to an underestimate of the velocity, and the solid fraction below this point.

To further confirm that the measurements were quantitative, the flow rate was measured gravimetrically and compared with the mass flow rate obtained from MRI in Figure 7. There is an excellent agreement between the integrated flow rate obtained from the MRI and the gravimetric measurement. The measured gravimetric mass flow rate was in fair agreement with previously published empirical correlations that relate the mass flow rate to the hopper angle and outlet size $[13,14]$. This finding confirms that our MRI measurements of the solid fraction and the velocity are quantitative.

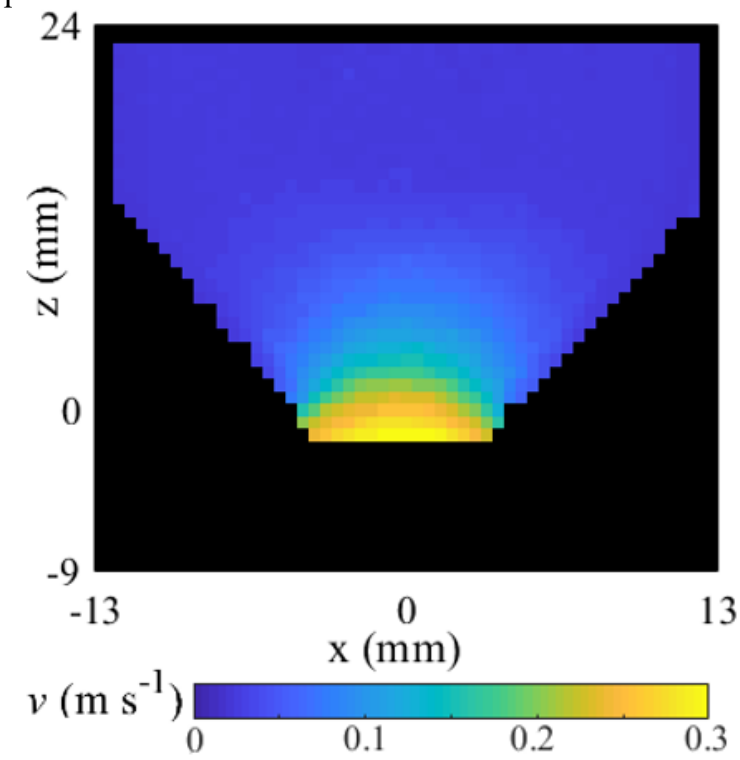

Fig. 6. The vertical 2D map of the vertical velocity.

\section{Conclusion}

It is necessary to have quantitative measurements of solid fraction and velocity to be able to characterize dense granular flows. Here, we showed that we are able to measure the solid fraction and velocity quantitatively under flow using a recently developed MRI technique. Excellent agreement was observed between the macroscopic mass flow rate obtained from the MRI and the gravimetric mass flow rate measurements, which confirms that our measurements are quantitative.

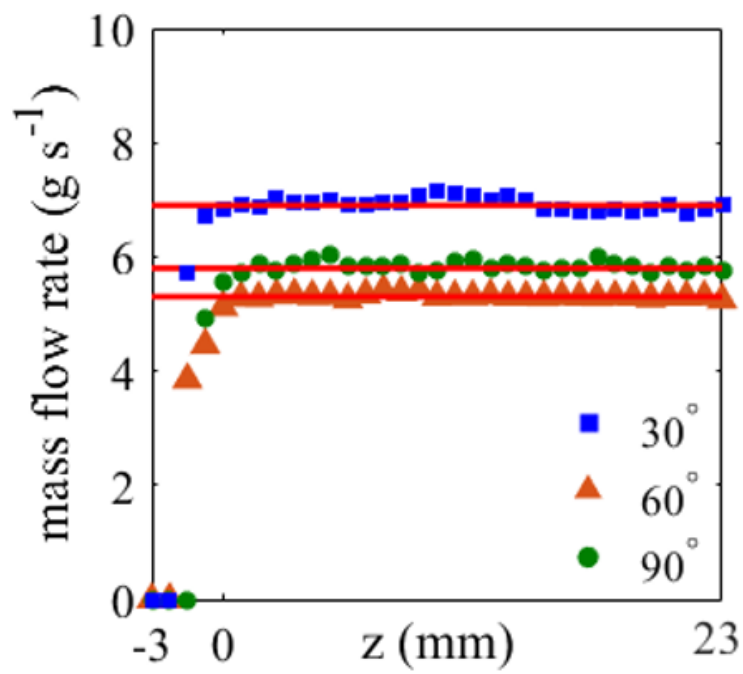

Fig. 7. MRI (symbols) and gravimetric (lines) mass flow rates.

\section{References}

[1] J.R. Darias, D. Gella, M.E. Fernández, I. Zuriguel, D. Maza, Powder Technol. 366, 488 (2020)

[2] M. Wang, Industrial Tomography: Systems and Applications (Woodhead Publishing, 2015)

[3] D.J. Parker, Rev. Sci. Instrum. 88, 051803 (2017)

[4] M. Danczyk, T. Meaclem, M. Mehdizad, D. Clarke, P. Galvosas, L. Fullard, D. Holland, Powder Technol. 372, 671 (2020).

[5] P. Moucheront, F. Bertrand, G. Koval, L. Tocquer, S. Rodts, J.N. Roux, A. Corfdir, F. Chevoir, Magn. Reson. Imaging. 28, 910 (2010)

[6] K. Sakaie, D. Fenistein, T.J. Carroll, M. Van Hecke, P. Umbanhowar, EPL, 84, 49902 (2008)

[7] M. Gentzler, G.I. Tardos, Chem. Eng. Sci. 64, 4463 (2009)

[8] D. Holland, Q. Marashdeh, C. Müller, F. Wang, J. Dennis, L.-S. Fan, L. Gladden, Ind. Eng. Chem. Res. 48, 172 (2008)

[9] M. Mehdizad, L. Fullard, P. Galvosas, D. Holland, J. Magn. Reson. 325, 106935 (2021)

[10] S. K. Bharti, R. Roy, Trends Anal. Chem. 35, 5 (2012)

[11] W.A. Beverloo, H.A. Leniger, J. De Van Velde, Chem. Eng. Sci. 15, 260 (1961)

[12] A.A. Khrapitchev, B. Newling, B.J. Balcom, J Magn. Reson. 178, 288 (2006)

[13] R. L. Brown, J. C. Richards, Rheol. Acta. 4, 153 (1965)

[14] H. Rose, T. Tanaka, Eng. 208, 465 (1959) 